\title{
Inverse Correlation between Testosterone and Ventricle Ejection Fraction, Hemodynamics and Exercise Capacity in Heart Failure Patients with Erectile Dysfunction
}

\author{
Edimar A. Bocchi, Vitor O. Carvalho, Guilherme V. Guimaraes \\ Laboratory of Heart Failure and Transplantation, Heart Institute, Incor, University of Sao Paulo, \\ SP, Brazil
}

\begin{abstract}
Background: Neurohormonal activation and abnormalities in growth hormone and testosterone concentrations have been reported in heart failure (HF). Erectile dysfunction(ED) is common in these patients and contributes to a low quality of life. No data are known regarding the correlation between testosterone and hemodynamics, exercise capacity and cardiac function in HF patients with ED, a marker of endothelial dysfunction. The aim of this study was to correlate testosterone levels with cardiac function, hemodynamic and exercise capacity in HF patients with ED.

Materials and Methods: Fifteen HF patients underwent a six-minute treadmill cardiopulmonary walking test (6'CWT) and, ten minutes later, a maximum cardiopulmonary exercise test. Also, testosterone and other hormones were determined at rest.

Results: Among hemodynamic variables only diastolic blood pressure on 6'CWT was correlated with testosterone levels(r $=-0.66, \mathrm{p}=0.007)$. The variables on exercise tests, $\mathrm{VE} / \mathrm{VCO}_{2}$ slope and oxygen consumption did not show any correlation, except the distance at 6'CWT $(\mathrm{r}=0.50, \mathrm{p}=0,047)$. Right and left ventricle ejection fraction showed inverse correlation with testosterone ( $\mathrm{r}=-0.55, \mathrm{p}=0.03$ and $\mathrm{r}=-0.69, \mathrm{p}=0.004$ respectively).

Conclusion: Testosterone levels correlated directly with distance at six-minute cardiopulmonary walk test and inversely with diastolic blood pressure, right and left ventricle ejection fraction in heart failure patients with erectile dysfunction. Further elucidation of mechanisms as regards testosterone action in these patients is warranted.
\end{abstract}

Key words: heart failure; hemodynamics; physical activity; testosterone; erectile dysfunction Int Braz J Urol. 2008; 34: 302-12

\section{INTRODUCTION}

Heart failure (HF) can be considered as the last stage of heart disease and a significant cause of mortality and morbidity worldwide (1). The left ventricular systolic dysfunction and limited exercise capacity manifested by breathlessness and fatigue are determinants of mortality and clinical events in the follow-up of HF patients. $(2,3)$. Multiple mechanisms have been reported to be related to exercise capacity including diastolic and systolic cardiac function, reflex, metabolic, vascular and muscular response (3). Sexual satisfaction is an important component that influences quality of life in HF patients. Erectile dysfunction (ED) affects 60 to $70 \%$ of HF clinic outpatients (4). Symptoms, hormonal abnormalities, hemodynamic status, medication side effects and psychological factors are the major contributors to this sexual disorder. 
In the physiopathology of chronic HF, the neurohormonal hypothesis for progression of heart failure has been considered of greater interest than the original hemodynamic mechanisms (5). Activation of sympathetic nervous system, renin-angiotensin-aldosterone system, arginine vasopressin, and endothelins are considered as neurohormonal targets in the treatment of HF. However, other hormonal abnormalities have been reported in HF such as significant decrease in growth hormones, insulin-like growth factor I and testosterone concentrations (6). The hormonal and cytokine activation contributes to peripheral muscle tissue wasting as well as anabolic/catabolic imbalance (7). In men, testosterone seems to play a role in determining anabolic function, anti-inflammatory and vasodilator processes. No data has been reported as regards testosterone's effect on hemodynamics, cardiac function and exercise capacity in HF patients with ED.

The aim of this study was to evaluate the correlation between serum testosterone levels and cardiac function, hemodynamics, and exercise capacity in $\mathrm{HF}$ patients with ED. In addition, we determined a HF hormone profile: serum levels of prolactin, luteinizing hormone, follicle-stimulating hormone, resting norepinephrine, and thyroid hormones.

\section{MATERIALS AND METHODS}

\section{Studied Population}

Fifteen randomized chronic male HF patients, $50 \pm 10$ years with ED (Table-1) for at least four months, in a steady relationship and presenting interest in sex were included. ED was defined as the inability to achieve or maintain a durable erection to permit satisfactory sexual intercourse (the 5 th-item of the International Index of Erectile Dysfunction) $(8,9)$. Patients were in stable clinical condition for three months without testosterone replacement or drugs that could have affected testosterone levels, as finasteride, opiates, glucocorticoids and anticonvulsants. All patients were sedentary and did not have heavy alcohol consumption, nephrotic syndrome or liver cirrhosis history. All patients were evaluated for ED etiology such as arterial insufficiency, venous leakage or penile fibrosis. No etiology was found, except HF syndrome. The protocol was approved by the Ethical Committee of the Heart Institute. Subjects provided written informed consent before participation. Exclusion criteria: ED secondary to causes other than HF, previous ED therapy, recent use of phosphodiesterase inhibitors, psychiatric or psychological disorders, unstable angina or recent myocardial infarction, syncope, high-risk arrhythmias, disease with limitation for exercise except HF, and symptomatic hypotension or systolic blood pressure (SBP) $<85 \mathrm{~mm} \mathrm{Hg}$.

\section{Exercise Protocol}

We measured systolic blood pressure (SBP) and diastolic blood pressure (DBP) with the patient in an upright position immediately before each exercise test, at the last minute of the six-minute walking test (6'WT), at maximum exercise peak, and at 1-minute recovery (10). Electrocardiography was continuously monitored. Pulmonary ventilation and gas exchange data were determined on a breathby-breath basis with a computerized system (model Vmax 229 Sensormedics). The six-minute walking test (6'WT) was performed using a programmable treadmill without inclination and with patient-controlled speed (Series 2000, Marquette Electronics) at least 2 hours after a light meal and with controlled room temperature $\left(21^{\circ} \mathrm{C}\right.$ to $\left.23^{\circ} \mathrm{C}\right)$. The patients were oriented to walk according to Borg's scale, with exertion level ranging from light to somewhat hard, from 11 to 13. After return of heart rate (HR), SBP, DBP, and symptoms to basal condition, patients underwent a progressive exercise test using a modified Naughton protocol. They were encouraged to perform maximum exercise until exhaustion or the onset of non tolerated symptoms occurred and the respiratory exchange ratio exceeded 1.0. The peak oxygen consumption (peak $\mathrm{VO}_{2}$ ) was considered the maximum reached $\mathrm{VO}_{2}$ value.

\section{Cardiac Function and Hormonal Determinations}

The right ventricular and left ventricular ejection fraction (RVEF and LVEF, respectively), as a percentage, were determined by echocardiogram. Hormonal dosage at rest before the exercise test included determination of serum total testosterone (fluoroimmunoassay method), prolactin, luteinizing 
Table 1 - Characteristics of patients with erectile dysfunction.

\begin{tabular}{lc}
\hline Characteristics of Heart Failure Patients & N of Patients (\%) or Value \\
\hline Etiology & 22 \\
$\quad$ Ischemic & 22 \\
$\quad$ Chagasic & 26 \\
$\quad$ Idiopathic dilated cardiomyopathy & 26 \\
$\quad$ Hypertensive & 4 \\
$\quad$ Valvular & \\
NYHA functional class & 83 \\
$\quad$ II & 13 \\
$\quad$ III & 4 \\
$\quad$ IV & 91 \\
Diuretics & 67 \\
ACE inhibitor & 8 \\
Angiotensin II AT1 receptor antagonists & 61 \\
B-Adrenergic receptor blocker & 61 \\
Spironolactone & 17 \\
Amiodarone & 9 \\
Isosorbide 5-mononitrate (40 mg) & $203 \pm 49$ \\
Total cholesterol, mg/dL & $46 \pm 13$ \\
HDL cholesterol, mg/dL & $130 \pm 41$ \\
LDL cholesterol, mg/dL & $155 \pm 145$ \\
Triglycerides, mg/dL & $24 \pm 19$ \\
Duration of ED symptoms, months & $61 \pm 49$ \\
Duration of CHF symptoms, months & \\
\hline
\end{tabular}

Values are mean $\pm S D$ or $n(\%) ; A C E=$ angiotensin-converting enzyme inhibitor, $C H F$-symptoms = congestive heart failure; ED = erectile dysfunction.

hormone, norepinephrine and follicle stimulating hormone. The normal value of testosterone in this study was 200-950 ng/dL. Lipid profile assessment was total cholesterol, HDL cholesterol, LDL cholesterol, Triglycerides. Due to hormonal circadian rhythms, all these tests were performed in the same period (early morning).

\section{Statistical Analysis}

The descriptive analysis was presented as mean and standard deviation. The variables studied underwent the non-parametric Spearman test for correlation, considering $\mathrm{p}<0.05$ (SPSS Statistical
Software for Windows version 11.5. Inc., Chicago, IL, USA).

\section{RESULTS}

All patients had total testosterone serum levels in the range for normal subjects $(200-950 \mathrm{ng} / \mathrm{dL})$. Four patients had prolactin serum levels below the normal range $(2.5-11.5 \mathrm{ng} / \mathrm{mL})$. One patient had hypothyroidism and the other hyperthyroidism. The cardiac function, left ventricular diameters, and hormonal values of patients are reported in Table-2. Table-3 shows the hemodynamic and exercise variables. 
Table 2 - Cardiac function, left ventricular diameters and hormonal values in patients with erectile dysfunction.

\begin{tabular}{lc}
\hline Variables of Heart Failure Patients & Number of Patients or Value \\
\hline LV ejection fraction (echo) \% & $23 \pm 7$ \\
RV ejection Fraction (echo) \% & $28 \pm 8$ \\
LV end-diastolic diameter (echo), mm & $72 \pm 16$ \\
LV end systolic diameter (echo), mm & $58 \pm 55$ \\
Hypothyroidism/hyperthyroidism & 2 patients \\
Serum total testosterone, ng/dL (normal 200-950) & $604 \pm 203$ \\
Prolactin, ng/mL (normal 2.5-11.5) & $4.2 \pm 2.3$ \\
Luteinizing hormone, IU/L (normal 1.4-9.2) & $3.6 \pm 1.9$ \\
Follicle-stimulating hormone, IU/L (normal 1-12) & $4.2 \pm 2.8$ \\
Resting norepinephrine, pg/mL (normal 40-268) & $448 \pm 214$ \\
\hline
\end{tabular}

Values are mean $\pm S D$ or $n(\%) ; L V=$ left ventricular; $R V=$ right ventricular.

Table 3 - Hemodynamic and exercise variables.

\begin{tabular}{|c|c|}
\hline Variable & p Value \\
\hline $\mathrm{HR} 6 \mathrm{~m}$ & $111 \pm 105 \mathrm{bpm}$ \\
\hline SBP 6m & $141 \pm 133 \mathrm{mmHg}$ \\
\hline DBP $6 m$ & $74 \pm 71 \mathrm{mmHg}$ \\
\hline HR max & $134 \pm 127 \mathrm{bpm}$ \\
\hline SBP max & $138 \pm 128 \mathrm{mmHg}$ \\
\hline DBP max & $78 \pm 74 \mathrm{mmHg}$ \\
\hline Distance $6 \mathrm{~m}$ & $0.20 \pm 0.03$ miles \\
\hline Slope $6 \mathrm{~m}$ & $31.7 \pm 7$ \\
\hline $\mathrm{VO}_{2} 6 \mathrm{~m}$ & $11.9 \pm 2.9 \mathrm{~mL} / \mathrm{Kg} / \mathrm{min}$ \\
\hline Slope Max & $34.3 \pm 8.7$ \\
\hline $\mathrm{VO}_{2} \mathrm{Max}$ & $17.8 \pm 3.9 \mathrm{~mL} / \mathrm{Kg} / \mathrm{min}$ \\
\hline Time Max & $12.3 \pm 8 \mathrm{~min}$ \\
\hline \multicolumn{2}{|c|}{ 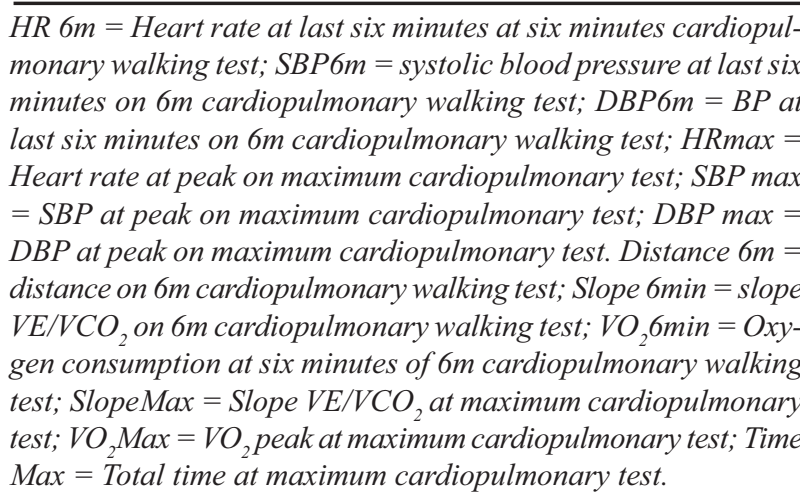 } \\
\hline
\end{tabular}

\section{Correlation between Testosterone Serum Levels and Cardiac Diameters, Cardiac Function, and Hemodynamic Data}

Left ventricle ejection fraction $(r=-0.69, p=$ 0.004) (Figure-1) and right ventricle ejection fraction $(\mathrm{r}=-0.55, \mathrm{p}=0.03)$ (Figure-1) showed inverse correlation with testosterone levels. SBP, DBP and heart rate during $\mathrm{MCT}$ (maximum cardiopulmonary test) did not show any correlation with testosterone levels or any hormonal serum levels except for DBP during the $6^{\prime} \mathrm{WT}$ that correlated with testosterone $(\mathrm{r}=-0.66$, $\mathrm{p}=0.007)$ (Figure-2). Left ventricular end diastolic diameter and left ventricular end systolic diameter did not show any correlation with testosterone levels either (Table-4)

\section{Correlation between Hormonal Levels and Exercise Capacity Data}

Exercise capacity variables on $6^{\prime} \mathrm{CWT}$, $\mathrm{VE} / \mathrm{VCO}_{2}$ slope at six minutes, oxygen consumption at six minutes did not any show correlation with testosterone levels or hormonal serum levels. Only maximum distance at $6^{\prime} \mathrm{CWT}$ showed correlation $(\mathrm{r}$ $=0.50, \mathrm{p}=0.047)$ with testosterone levels. Also during the $\mathrm{MCT}$, maximum $\mathrm{VE} / \mathrm{VCO}_{2}$ slope, maximum oxygen consumption and time of maximum test did not show correlation either (Table-4) 
A

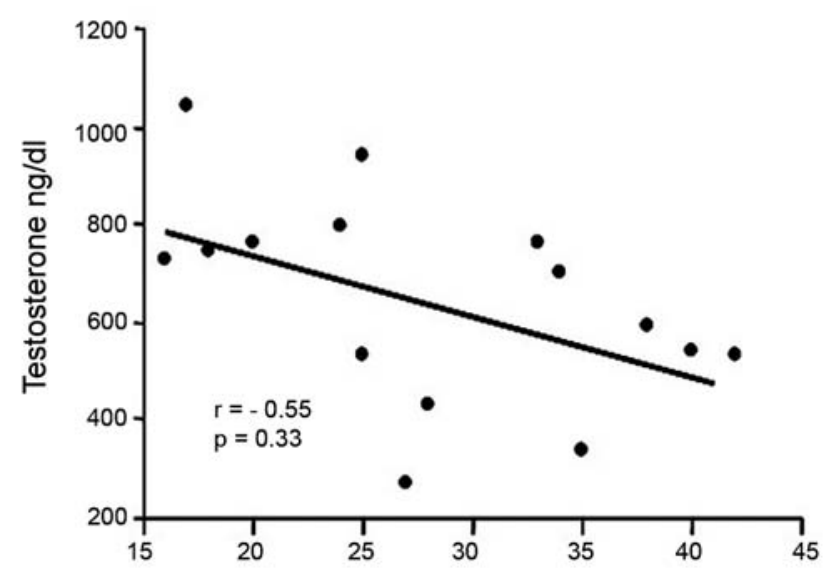

Ejection Fraction of Right Ventricle \% (By Echo)
B

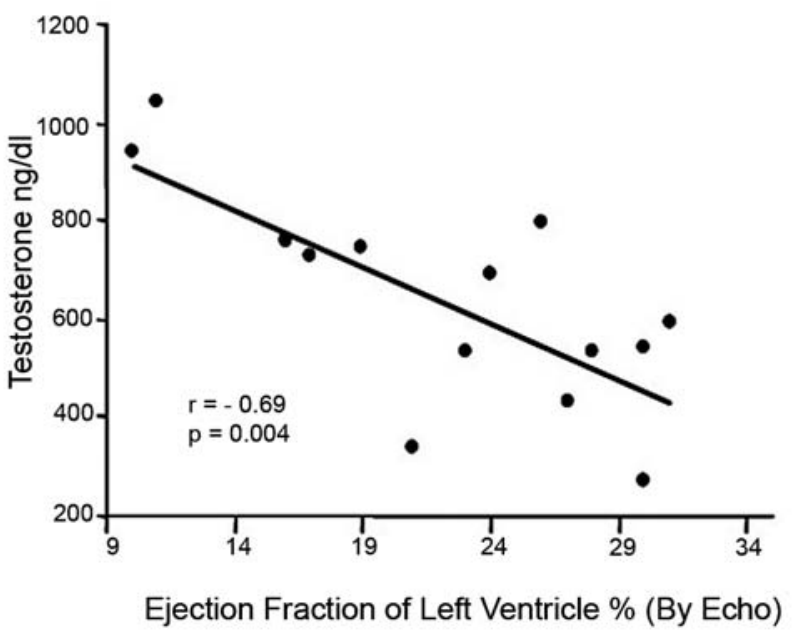

Figure 1 - A) Regression linear plots in all patients between total testosterone and right ventricular ejection fraction (\%, by echography). B) Regression linear plots in all patients between total testosterone serum levels and left ventricular ejection fraction (\%, by echography).

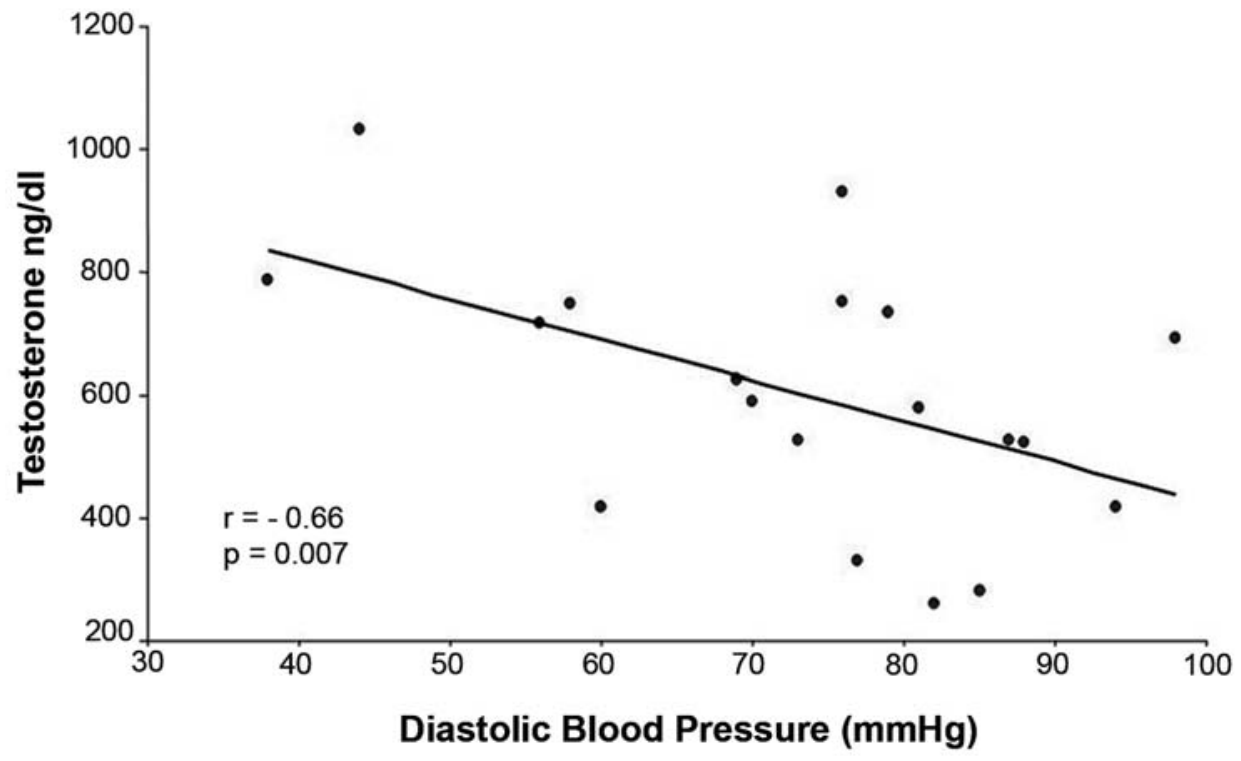

Figure 2 - Regression linear plots in all patients between total testosterone serum levels systemic diastolic blood pressure at the last minute of the 6 minutes walking test. 
Table 4 - Linear regression results between total testosterone serum levels and cardiac function, and exercise variables.

\begin{tabular}{lcc}
\hline Variable & r & p Value \\
\hline LVEF (in \%) & -0.69 & 0.004 \\
RVEF (in \%) & -0.55 & 0.03 \\
LVEDD (in mm) & -0.02 & $\mathrm{~ns}$ \\
LVESD (in mm) & 0.04 & $\mathrm{~ns}$ \\
HR 6m & 0.10 & $\mathrm{~ns}$ \\
SBP 6m & 0.16 & $\mathrm{~ns}$ \\
DBP 6m & -0.66 & 0.007 \\
HR max & -0.04 & $\mathrm{~ns}$ \\
SBP max & 0.16 & $\mathrm{~ns}$ \\
DBP max & -0.14 & $\mathrm{~ns}$ \\
Distance 6m & 0.50 & 0.047 \\
Slope 6m & -0.11 & $\mathrm{~ns}$ \\
VO 6m & 0.24 & $\mathrm{~ns}$ \\
Slope Max & -0.21 & $\mathrm{~ns}$ \\
VO Max & 0.02 & $\mathrm{~ns}$ \\
Time Max & 0.00 & $\mathrm{~ns}$ \\
\hline
\end{tabular}

$L V E F=$ Means left ventricular ejection fraction; $R V E F=$ Right ventricular $E F ; L V E D D=L V$ end diastolic diameter; $L V E S D=$ $L V$ end systolic diameter; HR $6 \mathrm{~m}=$ Heart rate at last six minutes at six minutes cardiopulmonary walking test; SBP6m = systolic blood pressure at last six minutes on $6 m$ cardiopulmonary walking test; DBP6m = BP at last six minutes on $6 \mathrm{~m}$ cardiopulmonary walking test; HRmax $=$ Heart rate at peak on maximum cardiopulmonary test; SBP max $=S B P$ at peak on maximum cardiopulmonary test; $D B P$ max $=D B P$ at peak on maximum cardiopulmonary test; Distance $6 \mathrm{~m}=$ distance on $6 \mathrm{~m}$ cardiopulmonary walking test; Slope $6 \mathrm{~min}=$ Slope $\mathrm{VE} / \mathrm{VCO}_{2}$ on $6 \mathrm{~m}$ cardiopulmonary walking test; $\mathrm{VO}, 6 \mathrm{~min}=$ Oxygen consumption at six minutes of $6 \mathrm{~m}$ cardiopulmonary walking test; SlopeMax = Slope $\mathrm{VE} / \mathrm{VCO}_{2}$ at maximum cardiopulmonary test; $\mathrm{VO}_{2}$ Max $=$ $\mathrm{VO}_{2}$ peak at maximum cardiopulmonary test; Time Max = total time at maximum cardiopulmonary test.

\section{COMMENTS}

Ours results demonstrated that there was an inverse correlation between right and left ventricular function and total testosterone serum levels in patients with ED. Other hemodynamic and exercise variables did not show a correlation with testosterone serum levels, except diastolic blood pressure and distance on 6'CWT. Other hormones abnormalities can be found in heart failure patients with erectile dysfunction.

Low testosterone levels were reported in severe stroke and acute myocardial infarction (11). In $\mathrm{HF}$, the testosterone serum levels may be low or in the normal range depending of the severity of the disease (11). Also, it has been reported that plasma levels of dehydroepiandrosterone sulfate decreased in patients with chronic HF in proportion to the severity evaluated by marker of cardiac function (12). However, to our knowledge this is the first time that an inverse correlation was demonstrated between left and right systolic cardiac function and total testosterone serum levels in selected patients with $\operatorname{HF}$ and $\operatorname{ED}(6,13,14)$. It is also partially discordant that, after testosterone administration, serum total levels remained in the normal physiological range and no significant changes were found in BNP, TNF- $\alpha$ or LVEF. Also, there was also a positive correlation between testosterone and cardiac output (15). However, it was concordant with worsening of left ventricular remodeling with testosterone administration. The mechanisms to explain our findings are not clear. Effects of testosterone in the heart are controversial and alternative hypotheses could be proposed.

The first hypothesis is that the total testosterone serum blood levels could play a role in pathophysiology of HF, worsening cardiac function. This is concordant with the concept that anabolic steroids were considered as having cardiac toxicity with alterations of cellular pathology and organ physiology similar to those seen with heart failure and cardiomyopathy (16). In addition, testosterone treatment in very high supra-physiological doses causes myocardial hypertrophy and stiffening (17). Investigators administrated physiologic doses of testosterone and found an increase in the left ventricular diameters, but did not find any change in the LVEF (18).

The second hypothesis, in contrast is that testosterone serum levels could have beneficial effects on HF, and a resistance for testosterone could be proposed in selected HF patients (6). In rats, androgen therapy has been reported to improve coronary blood flow and increased both fractional shortening, thereby improving cardiac function. In addition, animal stud- 
ies demonstrated vasodilator effects of androgens with potential beneficial effects in endothelial dysfunction (19), increment in IGF-1 levels with reduction in hyperinsulinemia and insulin resistance (20). In addition, resistance to growth hormone $(\mathrm{GH})$ was also proposed in severe HF patients based on higher GH concentrations in proportion with low IGF-1 concentration (21). However, the hypothesis of testosterone resistance needs to be proved in future investigations including mediators, nongenomic and genomic androgen action mechanisms (10).

Our method to include these patients in this study could have influenced our results because of specific factors related to erectile dysfunction in HF and higher severity of our patients in comparison with other studies (18). Patients with HF may experience erectile dysfunction for similar reasons to the general population, however, there are social, psychological, physiological, and drug-related consequences specific to HF (22). However, the influence of ED in our patients should be considered if the concept that this symptom is a marker of severity of HF and endothelial dysfunction is accepted (23). Endothelial dysfunction appears to affect all cardiac and peripheral circulation. Significant relationship between sexual performance and functional class and six-minute walk test has been reported (24). Moreover, as suggested for GH resistance in more severe patients, our results could have been influenced by these factors.

The correlation between total testosterone and exercise capacity is concordant with previous randomized, double blind, placebo-controlled parallel trial of testosterone replacement therapy. Exercise capacity significantly improved with testosterone therapy, compared with placebo (18). There are evidences in animal studies that anabolic androgens attenuate muscle fatigue in response to exercise, although the mechanism has not been identified (25). In addition, androgens may have different effects on heart and peripheral muscle (15).

Our correlation between testosterone and exercise, systemic DBP is in concordance with vasodilator effect of testosterone, but previous reports have speculated that for a vasodilatation effect of testosterone supra physiological concentrations are required $(26,27)$. However, beyond the vessel relaxation testosterone effect, this may activate the renin-angiotensin system $(10,28)$. Also, vascular effects could be dose-dependent manner, with opposite effects according to the dose.

Despite previous reported improvement in New York Heart Association functional class and exercise capacity, the potential prescription of testosterone for HF should be evaluated. This potential prescription should consider our results and previous study that showed the increment of left diameters in HF patients after testosterone administration (17). Further investigations should be performed concerning elucidation of its action in the heart and to determine if it is safe. Acute hemodynamics, exercise and functional beneficial effects cannot guarantee a short and long-term benefit in cardiac function and primary endpoints for heart failure treatment $(17,27)$.

Although this study is limited by the number of patients and did not include other markers of HF, nevertheless cardiac function could be considered as one of the main surrogate endpoints in heart failure. A better elucidation of testosterone mechanisms and action is warranted in a larger patient population.

\section{CONCLUSION}

Testosterone levels correlated directly with distance at six-minute cardiopulmonary walk test and inversely with diastolic blood pressure, right and left ventricle ejection fraction in heart failure patients with erectile dysfunction.

\section{CONFLICT OF INTEREST}

None declared.

\section{REFERENCES}

1. Bocchi EA, Vilas-Boas F, Perrone S, Caamaño AG, Clausell N, Moreira M da C, Thierer J, et al.: I Latin American Guidelines for the Assessment and Management of Decompensated Heart Failure. Arq Bras Cardiol. 2005; 85 (Suppl 3): 49-94. 
2. Olsson LG, Swedberg K, Ducharme A, Granger $\mathrm{CB}$, Michelson EL, McMurray JJ, et al.: Atrial fibrillation and risk of clinical events in chronic heart failure with and without left ventricular systolic dysfunction: results from the Candesartan in Heart failure-Assessment of Reduction in Mortality and morbidity (CHARM) program. J Am Coll Cardiol. 2006; 47: 1997-2004.

3. Harrington D, Anker SD, Chua TP, Webb-Peploe KM, Ponikowski PP, Poole-Wilson PA, et al.: Skeletal muscle function and its relation to exercise tolerance in chronic heart failure. J Am Coll Cardiol. 1997; 30: 1758-64.

4. Jaarsma T, Dracup K, Walden J, Stevenson LW: Sexual function in patients with advanced heart failure. Heart Lung. 1996; 25: 262-70.

5. R Ferrara, F Mastrorilli, G Pasanisi, S Censi, N D'aiello, A Fucili, et al.: Neurohormonal modulation in chronic heart failure. Eur Heart J. 2002; 4 (suppl D): D3-D11.

6. Kontoleon PE, Anastasiou-Nana MI, Papapetrou PD, Alexopoulos G, Ktenas V, Rapti AC, et al.: Hormonal profile in patients with congestive heart failure. Int J Cardiol. 2003; 87: 179-83.

7. Berry C, Clark ALÑ: Catabolism in chronic heart failure. Eur Heart J. 2000; 21: 521-32.

8. Wagner G, Saenz de Tejada I: Update on male erectile dysfunction. BMJ. 1998; 316: 678-82.

9. Rosen RC, Cappelleri JC, Smith MD, Lipsky J, Peña BM: Development and evaluation of an abridged, 5-item version of the International Index of Erectile Function (IIEF-5) as a diagnostic tool for erectile dysfunction. Int J Impot Res. 1999; 11: 319-26.

10. Bocchi EA, Guimarães G, Mocelin A, Bacal F, Bellotti G, Ramires JF: Sildenafil effects on exercise, neurohormonal activation, and erectile dysfunction in congestive heart failure: a doubleblind, placebo-controlled, randomized study followed by a prospective treatment for erectile dysfunction. Circulation. 2002; 106: 1097-103.

11. Liu PY, Death AK, Handelsman DJ: Androgens and cardiovascular disease. Endocr Rev. 2003; 24: 313-40.

12. Moriyama Y, Yasue H, Yoshimura M, Mizuno Y, Nishiyama K, Tsunoda R, et al.: The plasma levels of dehydroepiandrosterone sulfate are decreased in patients with chronic heart failure in proportion to the severity. J Clin Endocrinol Metab. 2000; 85: $1834-40$.

13. Woolf PD, Hamill RW, McDonald JV, Lee LA, Kelly M: Transient hypogonadotropic hypogonadism caused by critical illness. J Clin Endocrinol Metab. 1985; 60: 444-50.

14. Jeppesen LL, Jørgensen HS, Nakayama H, Raaschou HO, Olsen TS, Winther K: Decreased serum testosterone in men with acute ischemic stroke. Arterioscler Thromb Vasc Biol. 1996; 16: 74954.

15. Tappler B, Katz M: Pituitary-gonadal dysfunction in low-output cardiac failure. Clin Endocrinol (Oxf). 1979; 10: 219-26.

16. Sullivan ML, Martinez CM, Gennis P, Gallagher EJ: The cardiac toxicity of anabolic steroids. Prog Cardiovasc Dis. 1998; 41: 1-15.

17. Karila TA, Karjalainen JE, Mäntysaari MJ, Viitasalo MT, Seppälä TA: Anabolic androgenic steroids produce dose-dependant increase in left ventricular mass in power atheletes, and this effect is potentiated by concomitant use of growth hormone. Int J Sports Med. 2003; 24: 337-43.

18. Malkin CJ, Pugh PJ, West JN, van Beek EJ, Jones $\mathrm{TH}$, Channer KS: Testosterone therapy in men with moderate severity heart failure: a doubleblind randomized placebo controlled trial. Eur Heart J. 2006; 27: 57-64.

19. Pugh PJ, English KM, Jones TH, Channer KS: Testosterone: a natural tonic for the failing heart? QJM. 2000; 93: 689-94.

20. Hobbs CJ, Plymate SR, Rosen CJ, Adler RA: Testosterone administration increases insulinlike growth factor-I levels in normal men. J Clin Endocrinol Metab. 1993; 77: 776-9.

21. Bocchi E, Moura L, Guimarães G, Conceição Souza GE, Ramires JA: Beneficial effects of high doses of growth hormone in the introduction and optimization of medical treatment in decompensated congestive heart failure. Int J Cardiol. 2006; 110: 313-7.

22. Rastogi S, Rodriguez JJ, Kapur V, Schwarz ER: Why do patients with heart failure suffer from erectile dysfunction? A critical review and suggestions on how to approach this problem. Int J Impot Res. 2005; 17 (Suppl 1): S25-36. 
23. Maguire SM, Nugent AG, McGurk C, Johnston GD, Nicholls DP: Abnormal vascular responses in human chronic cardiac failure are both endothelium dependent and endothelium independent. Heart. 1998; 80: 141-5.

24. Jaarsma T, Dracup K, Walden J, Stevenson LW: Sexual function in patients with advanced heart failure. Heart Lung. 1996; 25: 262-70.

25. Tamaki T, Uchiyama S, Uchiyama Y, Akatsuka A, Roy RR, Edgerton VR: Anabolic steroids increase exercise tolerance. Am J Physiol Endocrinol Metab. 2001; 280: E973-81.
26. Crews JK, Khalil RA: Antagonistic effects of 17 beta-estradiol, progesterone, and testosterone on $\mathrm{Ca} 2+$ entry mechanisms of coronary vasoconstriction. Arterioscler Thromb Vasc Biol. 1999; 19: 1034-40.

27. Pugh PJ, Jones TH, Channer KS: Acute haemodynamic effects of testosterone in men with chronic heart failure. Eur Heart J. 2003; 24: 909-15.

28. Khalil RA: Sex hormones as potential modulators of vascular function in hypertension. Hypertension. 2005; 46: 249-54. $\overline{\text { Accepted after revision: }}$

April 20, 2008

\section{Correspondence address:}

Dr. Vitor Oliveira Carvalho

Laboratório de ICC-Transplante

INCOR, HCFMUSP

Av. Dr. Enéas de Carvalho Aguiar

05403-000, Sao Paulo, SP, Brazil

Fax: + $55113069-5419$

E-mail: vitor.carvalho@usp.br

\section{EDITORIAL COMMENT}

Decreased testosterone level was reported throughout a 4-year follow-up in elderly patients with erectile dysfunction (ED) in addition to the association with an adverse metabolic profile (1). Testosterone deficiency is a common occurrence in men with chronic heart failure (CHF) and may underpin features of advanced disease, including reduced skeletal muscle mass and fatigue (2). Testosterone is known to act as a vasodilator in systemic, coronary and pulmonary vascular beds, as well as having anabolic properties (3). This effect could potentially lead to increased cardiac output and improved cardiovascular function that may contribute to the clinical benefit. Therefore, administration of testosterone to men with chronic congestive heart failure may lead to hemodynamic alterations. Furthermore, testosterone deficiency is positively correlated with cardiac output and exercise capacity in patients with $\mathrm{CHF}$, whereas a significant improvement in both these parameters has been observed following testosterone replacement therapy. Testosterone therapy has also been shown to reduce circulating levels of inflammatory markers, (TNF- $\alpha$ and IL-1 $\beta$ ) in patients with established coronary artery disease and testosterone deficiency (4).

Although the mechanisms are poorly understood, the improvement in exercise capacity was 
shown to be positively correlated with the increase in serum testosterone level and was accompanied by a small increase in internal left ventricular length (2). As testosterone is a vasodilator, this could explain its anti-ischemic effects on cardiac function during exercise. However, it is currently unknown whether the vasodilatory effects of testosterone can influence the fatigability of skeletal muscle in a similar fashion. Therefore, adjunctive testosterone therapy might augment the positive effects of exercise rehabilitation on these clinical outcomes in hypogonadal males with stable CHF.

Currently, the main drawbacks in the design of clinical protocols and the inclusion of patients for the study of hormonal alteration lie in establishing the best assay for $\mathrm{T}$ measurement and in defining a baseline T cut off level. Although some limitations of the current study have been mentioned by the authors, the important feature of this study is that it addresses one timely and important issue, which is the correlation between serum testosterone levels and cardiac function, hemodynamic, and exercise capacity in HF patients with ED.

\section{REFERENCES}

1. El-Sakka AI, Hassoba HM: Age related testosterone depletion in patients with erectile dysfunction. J Urol. 2006; 176: 2589-93.

2. Malkin CJ, Pugh PJ, West JN, van Beek EJ, Jones TH, Channer KS: Testosterone therapy in men with moderate severity heart failure: a double-blind randomized placebo controlled trial. Eur Heart J. 2006; 27: 57-64.

3. English KM, Jones RD, Jones TH, Morice AH, Channer KS: Testosterone acts as a coronary vasodilator by a calcium antagonistic action. J Endocrinol Invest. 2002; 25: 455-8.

4. Malkin CJ, Pugh PJ, Jones RD, Kapoor D, Channer KS, Jones TH: The effect of testosterone replacement on endogenous inflammatory cytokines and lipid profiles in hypogonadal men. J Clin Endocrinol Metab. 2004; 89: 3313-8.

Dr. Ahmed I. El-Sakka Suez Canal University School of Medicine Ismailia, Egypt E-mail:aielsakka@yahoo.com

Dr. Farid Saad Bayer Schering Pharma Scientific Affairs Men's Healthcare Berlin, Germany

\section{EDITORIAL COMMENT}

In this study Bocchi, and his colleagues documented that testosterone (TT) levels correlated directly with distance at six-minute cardiopulmonary walk test and inversely with diastolic blood pressure, right and left ventricle ejection fraction in heart failure patients with erectile dysfunction (ED). Erectile dysfunction is considered an early sign of endothelial dysfunction and hence cardiovascular disorders while low TT levels are related to ED.

TT level is considered a marker of vascular reactivity and non-traditional risk factor (Bio Marker) for coronary artery disease and peripheral arterial disease. It is also related to low arrhythmia threshold and prolonged QT intervals.

Low TT level is a predictor of cardiovascular mortality, and marker of low exercise tolerance and poor quality of life. So if used as an adjuvant in treatment of heart failure it may significantly improve the functional capacity and symptoms of heart failure.

Although the number of cases in this study is limited, as stated by the authors, the paper is interesting not only to the Urologist and Andrologists 
but also to the cardiologist and general physicians. However, $61 \%$ of men included in this study were taking spironolactone, which is an anti-androgen rendering interpretation of the data a little bit difficult. Understanding the correlation between TT and cardiac functions will have a great beneficial effect on ED patients with heart failure.
Further studies using cardiac speckle technique and tissue Doppler imaging can provide more accurate assessment of cardiac function and perhaps demonstrate a correlation between TT levels and cardiac function.

\author{
Dr. Ahmed Asaad \\ Cardiology Consultant \\ National Heart Institute \\ Cairo, Egypt \\ Dr. Wael Zohdy \\ Department of Andrology \\ Cairo University \\ Cairo, Egypt \\ E-mail:wzohdy62@hotmail.com
}

